\title{
MAXIMAL ZERO PRODUCT SUBRINGS AND INNER IDEALS OF SIMPLE RINGS
}

\author{
ALEXANDER BARANOV AND ANTONIO FERNÁNDEZ LÓPEZ
}

\begin{abstract}
Let $Q$ be a (not necessarily unital) simple ring or algebra. A nonempty subset $S$ of $Q$ is said to have zero product if $S^{2}=0$. We classify all maximal zero product subsets of $Q$ by proving that the map $\mathcal{R} \mapsto \mathcal{R} \cap \operatorname{LeftAnn}(\mathcal{R})$ is a bijection from the set of all proper nonzero annihilator right ideals of $Q$ onto the set of all maximal zero product subsets of $Q$. We also describe the relationship between the maximal zero product subsets of $Q$ and the maximal inner ideals of its associated Lie algebra.
\end{abstract}

\section{INTRODUCTION}

Let $Q$ be a (not necessarily unital) associative ring or algebra. A nonempty subset $S$ of $Q$ is said to have zero product if $S^{2}=0$. By Zorn's Lemma, any zero product subset is contained in a maximal one, which is obviously a zero product subring. Note also that 0 is the unique maximal zero product subset of a ring $Q$ if and only if $Q$ has no nonzero nilpotent elements.

In this paper we describe the maximal zero product subsets of a prime $\operatorname{ring} Q$ with nonzero heart, in particular, of a simple ring, by proving that the map $\mathcal{R} \mapsto \mathcal{R} \cap$ $\operatorname{LeftAnn}(\mathcal{R})$ is a bijection from the set of all proper nonzero annihilator right ideals of $Q$ onto the set of all maximal zero product subsets of $Q$. In particular, if $Q$ is a simple unital Baer ring (e.g. a simple Artinian ring), all maximal zero product subsets of $Q$ are of the form $e Q(1-e)$, where $e$ is a nontrivial idempotent of $Q$. Moreover, if $e_{1}$ and $e_{2}$ are idempotents of $Q$ then $e_{1} Q\left(1-e_{1}\right)=e_{2} Q\left(1-e_{2}\right)$ if and only if $e_{1} e_{2}=e_{2}$ and $e_{2} e_{1}=e_{1}$ (equivalently, $e_{1} Q=e_{2} Q$ ).

In the case when $Q$ is a simple ring coinciding with its socle, we classify the maximal zero product subsets of $Q$ in terms of the associated geometry.

Finally, we describe the relationship between the maximal zero product subsets of a simple ring and the inner ideal structure of its associated Lie algebra.

2010 Mathematics Subject Classification. 16D30, 17B60.

Supported by University of Leicester.

Supported by the Spanish MEC and Fondos FEDER, MTM2014-52470-P. 
For simplicity of exposition, all results in the paper are stated for rings, but it is easy to see that they also hold for algebras over a field.

\section{Preliminaries and nOtation}

Throughout the paper, $Q$ is a (not necessarily unital) associative ring (or algebra); $\mathcal{L}$ denotes a left and $\mathcal{R}$ a right ideal of $Q ; \mathcal{J}_{r}(Q)$ and $\mathcal{J}_{l}(Q)$ are the lattices of all right and left ideals of $Q$, respectively. By an ideal we mean a two-sided ideal.

2.1. For a nonempty subset $S$ of $Q$ we denote by

$$
\operatorname{lann}(S)=\operatorname{LeftAnn}(S):=\{a \in Q: a S=0\}
$$

the left annihilator of $S$. Note that $\operatorname{lann}(S)$ is a left ideal of $Q$ (an ideal if $S$ is a left ideal). A left ideal $\mathcal{L}$ is said to be an annihilator left ideal if $\mathcal{L}=\operatorname{lann}(S)$ for some nonempty subset $S$ of $Q$. Similarly, one defines the right annihilator $\operatorname{rann}(S):=\{a \in Q: S a=0\}$, which is called an annihilator right ideal. Note that $\operatorname{lann}(S)=\operatorname{lann}(T)$ where $T=$ $S+S Q$ is the right ideal of $Q$ generated by $S . \operatorname{Similarly,} \operatorname{rann}(S)=\operatorname{rann}(S+Q S)$.

2.2. A ring $Q$ is said to be semiprime if $I^{2}=0$ implies $I=0$ for any ideal $I$ of $Q$; equivalently, $a Q a=0$ implies $a=0$ for every $a \in Q$. If $Q$ is semiprime, then $\operatorname{lann}(I)=\operatorname{rann}(I)$ and $I \cap \operatorname{rann}(I)=0$ for any ideal $I$ of $Q$.

2.3. A ring $Q$ is said to be prime if $I J=0$ implies $I=0$ or $J=0$ for $I, J$ ideals of $Q$. For a ring $Q$ the following conditions are equivalent:

(i) $Q$ is prime.

(ii) $\operatorname{lann}(I)=0$ for any nonzero ideal $I$ of $Q$.

(iii) $a I b=0$ implies $a=0$ or $b=0$, for any nonzero ideal $I$ of $Q$ and any $a, b$ in $Q$.

2.4. Let $Q$ be a ring. The heart of $Q$, denoted by heart $(Q)$, is defined as the intersection of all nonzero ideals of $Q$. Clearly, if $Q$ is simple then heart $(Q)=Q$. If $Q$ has nonzero heart, then heart $(Q)$ is a minimal ideal. Moreover, a prime ring has nonzero heart if and only if it contains a minimal ideal. If $Q$ is prime with nonzero $\operatorname{socle} \operatorname{soc}(Q)$ (the sum of all minimal left ideals), then by [8, Theorem III.3.1], $\operatorname{soc}(Q)$ is a simple ideal of $Q$, contained in any nonzero ideal of $Q$, so heart $(Q)=\operatorname{soc}(Q)$.

\section{ORThogonal PAIRS OF ONE-SIDED IDEALS}

3.1. We have a Galois connection between the lattice $\mathcal{J}_{r}(Q)$ of all right ideals of $Q$ and the lattice $\mathcal{J}_{l}(Q)$ of all left ideals of $Q$ given by $\mathcal{R} \mapsto \operatorname{lann}(\mathcal{R})$ and $\mathcal{L} \mapsto \operatorname{rann}(\mathcal{L})$, that is, 
(i) $\mathcal{L}_{1} \subseteq \mathcal{L}_{2} \Rightarrow \operatorname{rann}\left(\mathcal{L}_{2}\right) \subseteq \operatorname{rann}\left(\mathcal{L}_{1}\right)$ and $\mathcal{R}_{1} \subseteq \mathcal{R}_{2} \Rightarrow \operatorname{lann}\left(\mathcal{R}_{2}\right) \subseteq \operatorname{lann}\left(\mathcal{R}_{1}\right)$,

(ii) $\mathcal{L} \subseteq \operatorname{lann}(\operatorname{rann}(\mathcal{L}))$ and $\mathcal{R} \subseteq \operatorname{rann}(\operatorname{lann}(\mathcal{R}))$,

for all $\mathcal{L}, \mathcal{L}_{1}, \mathcal{L}_{2} \in \mathcal{J}_{l}(Q)$ and $\mathcal{R}, \mathcal{R}_{1}, \mathcal{R}_{2} \in \mathcal{J}_{r}(Q)$.

Denote by $\overline{\mathcal{L}}:=\operatorname{lann}(\operatorname{rann}(\mathcal{L}))$ and $\overline{\mathcal{R}}:=\operatorname{rann}(\operatorname{lann}(\mathcal{R}))$ the corresponding closures of $\mathcal{L}$ and $\mathcal{R}$. We say that $\mathcal{L}$ (resp. $\mathcal{R}$ ) is closed if $\mathcal{L}=\overline{\mathcal{L}}$ (resp. $\mathcal{R}=\overline{\mathcal{R}}$ ). It follows from (i) and (ii) that

$$
\operatorname{rann}(\mathcal{L}) \subseteq \overline{\operatorname{rann}(\mathcal{L})}=\operatorname{rann}(\operatorname{lann}(\operatorname{rann}(\mathcal{L})))=\operatorname{rann}(\overline{\mathcal{L}}) \subseteq \operatorname{rann}(\mathcal{L})
$$

and similarly for $\operatorname{lann}(\mathcal{R})$. Therefore we have

(iii) $\operatorname{rann}(\mathcal{L})=\overline{\operatorname{rann}(\mathcal{L})}=\operatorname{rann}(\overline{\mathcal{L}})$,

(iv) $\operatorname{lann}(\mathcal{R})=\overline{\operatorname{lann}(\mathcal{R})}=\operatorname{lann}(\overline{\mathcal{R}})$.

In particular,

(v) a right (resp. left) ideal is closed if and only if it is an annihilator right (resp. left) ideal.

3.2. By an orthogonal pair of $Q$ we mean a pair $(\mathcal{R}, \mathcal{L})$, where $\mathcal{R}$ is a nonzero right and $\mathcal{L}$ is a nonzero left ideals of $Q$ such that $\mathcal{L} \mathcal{R}=0$.

Lemma 3.3. For an orthogonal pair $(\mathcal{R}, \mathcal{L})$ the following conditions are equivalent:

(i) $\mathcal{R}=\overline{\mathcal{R}}$ and $\mathcal{L}=\operatorname{lann}(\mathcal{R})$,

(ii) $\mathcal{R}=\operatorname{rann}(\mathcal{L})$ and $\mathcal{L}=\operatorname{lann}(\mathcal{R})$,

(iii) $\mathcal{L}=\overline{\mathcal{L}}$ and $\mathcal{R}=\operatorname{rann}(\mathcal{L})$.

Proof. We will prove (i) $\Leftrightarrow($ ii). The proof of (ii) $\Leftrightarrow($ iii) is similar. Suppose that $\mathcal{L}=$ $\operatorname{lann}(\mathcal{R})$. Then $\operatorname{rann}(\mathcal{L})=\operatorname{rann}(\operatorname{lann}(\mathcal{R})=\overline{\mathcal{R}}$, so that $\mathcal{R}=\overline{\mathcal{R}} \Leftrightarrow \mathcal{R}=\operatorname{rann}(\mathcal{L})$.

Given two orthogonal pairs $\left(\mathcal{R}_{1}, \mathcal{L}_{1}\right)$ and $\left(\mathcal{R}_{2}, \mathcal{L}_{2}\right)$, we say that $\left(\mathcal{R}_{1}, \mathcal{L}_{1}\right) \subseteq\left(\mathcal{R}_{2}, \mathcal{L}_{2}\right)$ if and only if $\mathcal{R}_{1} \subseteq \mathcal{R}_{2}$ and $\mathcal{L}_{1} \subseteq \mathcal{L}_{2}$. This gives a partial order on the set of orthogonal pairs.

Proposition 3.4. Let $(\mathcal{R}, \mathcal{L})$ be an orthogonal pair of $Q$. Then the following hold.

(i) $(\overline{\mathcal{R}}, \operatorname{lann}(\mathcal{R}))$ and $(\operatorname{rann}(\mathcal{L}), \overline{\mathcal{L}})$ are maximal orthogonal pairs.

(ii) $(\mathcal{R}, \mathcal{L})$ is contained in the maximal orthogonal pairs $(\overline{\mathcal{R}}, \operatorname{lann}(\mathcal{R}))$ and $(\operatorname{rann}(\mathcal{L}), \overline{\mathcal{L}})$.

(iii) $(\mathcal{R}, \mathcal{L})$ is maximal if and only if it satisfies the equivalent conditions of Lemma 3.3. 
Proof. (i) Since $\mathcal{R} \subseteq \overline{\mathcal{R}}$ and $\mathcal{L} \subseteq \operatorname{lann}(\mathcal{R})$, both $\overline{\mathcal{R}}$ and $\operatorname{lann}(\mathcal{R})$ are nonzero; and since $\operatorname{lann}(\mathcal{R})=\operatorname{lann}(\overline{\mathcal{R}})$, we have that $(\overline{\mathcal{R}}, \operatorname{lann}(\mathcal{R}))$ is an orthogonal pair. Suppose now that $(\overline{\mathcal{R}}$, lann $(\mathcal{R}))$ is contained in an orthogonal pair $\left(\mathcal{R}^{\prime}, \mathcal{L}^{\prime}\right)$. Then $\overline{\mathcal{R}} \subseteq \mathcal{R}^{\prime}$ implies $\operatorname{lann}\left(\mathcal{R}^{\prime}\right) \subseteq \operatorname{lann}(\overline{\mathcal{R}})=\operatorname{lann}(\mathcal{R})$, so

$$
\mathcal{L}^{\prime} \subseteq \operatorname{lann}\left(\mathcal{R}^{\prime}\right) \subseteq \operatorname{lann}(\mathcal{R}) \subseteq \mathcal{L}^{\prime}
$$

which proves that $\operatorname{lann}(\mathcal{R})=\mathcal{L}^{\prime}$. Hence

$$
\mathcal{R}^{\prime} \subseteq \operatorname{rann}\left(\mathcal{L}^{\prime}\right)=\overline{\mathcal{R}} \subseteq \mathcal{R}^{\prime},
$$

which proves that $\overline{\mathcal{R}}=\mathcal{R}^{\prime}$. Therefore the orthogonal pair $(\overline{\mathcal{R}}, \operatorname{lann}(\mathcal{R}))$ is maximal. Similarly, one can prove that $(\operatorname{rann}(\mathcal{L}), \overline{\mathcal{L}})$ is a maximal orthogonal pair.

(ii) As noted in the proof of $(\mathrm{i}),(\mathcal{R}, \mathcal{L})$ is contained in the maximal orthogonal pair $(\overline{\mathcal{R}}$, lann $(\mathcal{R}))$. Similarly, $(\mathcal{R}, \mathcal{L})$ is also contained in the maximal orthogonal pair $(\operatorname{rann}(\mathcal{L}), \overline{\mathcal{L}})$.

(iii) Suppose that $(\mathcal{R}, \mathcal{L})$ is maximal. Then $(\mathcal{R}, \mathcal{L}) \subseteq(\overline{\mathcal{R}}, \operatorname{lann}(\mathcal{R}))$ implies $\mathcal{R}=\overline{\mathcal{R}}$ and $\mathcal{L}=\operatorname{lann}(\mathcal{R})$.

Proposition 3.5. Let $B$ be an additive subgroup of $Q$. Then the following conditions are equivalent:

(i) $B Q B \subseteq B$ and $B^{2}=0$.

(ii) There exist $\mathcal{L} \in \mathcal{J}_{l}(Q)$ and $\mathcal{R} \in \mathcal{J}_{r}(Q)$ such that $\mathcal{R} \mathcal{L} \subseteq B \subseteq \mathcal{R} \cap \mathcal{L}$ and $\mathcal{L} \mathcal{R}=0$.

Proof. (i) $\Rightarrow$ (ii): Taking $\mathcal{L}=B+Q B$ and $\mathcal{R}=B+B Q$, it is easily seen that (ii) holds.

(ii) $\Rightarrow$ (i): Clearly, $B^{2} \subseteq \mathcal{L} \mathcal{R}=0$ and $B Q B \subseteq \mathcal{R} Q \mathcal{L} \subseteq \mathcal{R} \mathcal{L} \subseteq B$.

3.6. Following [1, we say that an additive subgroup $B$ of $Q$ is a regular inner ideal of $Q$ if it satisfies the equivalent conditions of the above proposition. In that case the orthogonal pair $(\mathcal{R}, \mathcal{L})$ in (ii) is said to be associated to $B$. We note the following properties of regular inner ideals.

(i) If $B$ is nonzero in Proposition 3.5 , then both $\mathcal{R}$ and $\mathcal{L}$ are nonzero and therefore $(\mathcal{R}, \mathcal{L})$ is an orthogonal pair.

(ii) If $Q$ is a prime ring and $(\mathcal{R}, \mathcal{L})$ is an orthogonal pair, then any additive subgroup $B$ of $Q$ with $\mathcal{R} \mathcal{L} \subseteq B \subseteq \mathcal{R} \cap \mathcal{L}$ is a nonzero regular inner ideal, since $B=0$ would imply $\mathcal{R} Q \mathcal{L} \subseteq \mathcal{R} \mathcal{L} \subseteq B=0$, which is a contradiction by 2.3(iii).

(iii) If $Q$ is a von Neumann regular ring, then any orthogonal pair $(\mathcal{R}, \mathcal{L})$ gives rise to a unique regular inner ideal $B=\mathcal{R} \mathcal{L}=\mathcal{R} \cap \mathcal{L}$, since

$$
\mathcal{R} \cap \mathcal{L} \subseteq(\mathcal{R} \cap \mathcal{L}) Q(\mathcal{R} \cap \mathcal{L}) \subseteq \mathcal{R} \mathcal{L} \subseteq \mathcal{R} \cap \mathcal{L}
$$




\section{ZERO PRODUCT SUBRINGS OF PRIME RINGS WITH NONZERO HEART}

The following lemma shows that the orthogonal pair associated to a nonzero regular inner ideal of a prime ring with nonzero heart (see 2.4) is defined almost uniquely.

Lemma 4.1. Let $Q$ be a prime ring with nonzero heart $H=\operatorname{heart}(Q)$ and let $B$ be a nonzero regular inner ideal of $Q$ with associated orthogonal pair $(\mathcal{R}, \mathcal{L})$. Then $B H=\mathcal{R} H$ and $H B=H \mathcal{L}$. In particular, if $Q$ is simple and unital, then $B Q=\mathcal{R}$ and $Q B=\mathcal{L}$.

Proof. By 2.3(iii), the ideal $\mathcal{L} H \mathcal{R}$ is nonzero. Since $\mathcal{L} H \mathcal{R} \subseteq H$ and $H$ is minimal, we have that $\mathcal{L} H \mathcal{R}=H$. Hence

$$
\mathcal{R} H=\mathcal{R} \mathcal{L} H \mathcal{R} \subseteq B H \mathcal{R}=B H \subseteq \mathcal{R} H,
$$

which proves that $B H=\mathcal{R} H$. Similarly, one proves that $H B=H \mathcal{L}$.

Lemma 4.2. Let $Q$ be a prime ring with nonzero heart $H$ and let $\left(\mathcal{R}_{1}, \mathcal{L}_{1}\right)$ and $\left(\mathcal{R}_{2}, \mathcal{L}_{2}\right)$ be maximal orthogonal pairs in $Q$. Then $\mathcal{R}_{1} \cap \mathcal{L}_{1} \subseteq \mathcal{R}_{2} \cap \mathcal{L}_{2}$ implies $\left(\mathcal{R}_{1}, \mathcal{L}_{1}\right)=\left(\mathcal{R}_{2}, \mathcal{L}_{2}\right)$.

Proof. Set $\mathcal{L}:=\mathcal{L}_{1}+\mathcal{L}_{2}$ and $B_{j}=\mathcal{R}_{j} \cap \mathcal{L}_{j}, j=1,2$. By Lemma 4.1 applied to $B_{1}$ and $B_{2}$, which are regular inner ideals by (3.6) (ii),

$$
H \mathcal{L}=H \mathcal{L}_{1}+H \mathcal{L}_{2}=H B_{1}+H B_{2} \subseteq H B_{2}=H \mathcal{L}_{2} \subseteq \mathcal{L}_{2} .
$$

We claim that $\mathcal{L R}_{2}=0$. Otherwise, $H=H \mathcal{L} \mathcal{R}_{2}$ (as $\mathcal{L} \mathcal{R}_{2}$ is a two-sided ideal and $H$ is minimal) and hence, by the formula displayed above,

$$
H=H \mathcal{L} \mathcal{R}_{2} \subseteq \mathcal{L}_{2} \mathcal{R}_{2}=0,
$$

which is a contradiction. Thus $\mathcal{L R}_{2}=0$ and hence

$$
\mathcal{L}_{1} \subseteq \mathcal{L} \subseteq \operatorname{lann}\left(\mathcal{R}_{2}\right)=\mathcal{L}_{2}
$$

by Proposition 3.4 (iii). Similarly, $\mathcal{R}_{1} \subseteq \mathcal{R}_{2}$. But then $\left(\mathcal{R}_{1}, \mathcal{L}_{1}\right)=\left(\mathcal{R}_{2}, \mathcal{L}_{2}\right)$ by maximality of $\left(\mathcal{R}_{1}, \mathcal{L}_{1}\right)$.

Now we are ready to prove our main result, which describes maximal zero product subsets of prime rings with nonzero hearts (in particular, of simple rings).

Theorem 4.3. Let $Q$ be a prime ring with nonzero heart containing nonzero nilpotent elements and let $S$ be a subset of $Q$. Then the following are equivalent.

(i) $S$ is a maximal zero product subset of $Q$.

(ii) $S$ is a maximal regular inner ideal of $Q$. 
(iii) $S=\mathcal{R} \cap \mathcal{L}$, where $(\mathcal{R}, \mathcal{L})$ is a maximal orthogonal pair, i.e. $\mathcal{R}=\operatorname{rann}(\mathcal{L})$ and $\mathcal{L}=\operatorname{lann}(\mathcal{R})$.

Proof. (i) $\Rightarrow$ (ii): Suppose that $S$ is a maximal zero product subset. Note that $S$ is nonzero as $Q$ contains nonzero nilpotent elements. Since span of $S$ is a zero product subset, $S$ is actually an additive subgroup of $Q$. Put $B=S Q S+S$. Then $S \subseteq B$ and $B^{2}=0$. Since $S$ is maximal, one has $S=B$. Therefore

$$
B Q B=S Q S \subseteq B
$$

so $B=S$ is a regular inner ideal of $Q$. Clearly, $B$ is maximal as $S$ is maximal.

(ii) $\Rightarrow$ (iii): Suppose that $S$ is a maximal regular inner ideal of $Q$. Note that $S$ is non-zero (otherwise, it is strictly contained in a non-zero maximal zero product subset $S^{\prime}$ of $Q$, which is a regular inner ideal of $Q$ as $\left.(\mathrm{i}) \Rightarrow(\mathrm{ii})\right)$. By Proposition 3.5, there is an orthogonal pair $(\mathcal{R}, \mathcal{L})$ such that $S \subseteq \mathcal{R} \cap \mathcal{L}$. By Proposition 3.4(ii), one can assume that the pair $(\mathcal{R}, \mathcal{L})$ is maximal. By Proposition $[3.5, \mathcal{R} \cap \mathcal{L}$ is a regular inner ideal of $Q$, so $S=\mathcal{R} \cap \mathcal{L}$ as $S$ is maximal.

(iii) $\Rightarrow(\mathrm{i})$ : Let $S=\mathcal{R} \cap \mathcal{L}$ where $(\mathcal{R}, \mathcal{L})$ is a maximal orthogonal pair. Then $S^{2} \subseteq$ $\mathcal{L} \mathcal{R}=0$, so $S$ is a zero product subset. Let $S^{\prime}$ be a maximal zero product subset of $Q$ containing $S$. By the implication (i) $\Rightarrow$ (iii), already established, $S^{\prime}=\mathcal{R}^{\prime} \cap \mathcal{L}^{\prime}$ where $\left(\mathcal{R}^{\prime}, \mathcal{L}^{\prime}\right)$ is a maximal orthogonal pair. By Lemma $4.2,(\mathcal{R}, \mathcal{L})=\left(\mathcal{R}^{\prime}, \mathcal{L}^{\prime}\right)$, so $S=S^{\prime}$, as desired.

Corollary 4.4. Let $Q$ be as in Theorem 4.3. Then the map $\mathcal{R} \mapsto \mathcal{R} \cap \operatorname{lann}(\mathcal{R})$ (resp. $\mathcal{L} \mapsto \mathcal{L} \cap \operatorname{rann}(\mathcal{L}))$ is a bijection from the set of all proper nonzero annihilator right (resp. left) ideals of $Q$ onto the set of all maximal zero product subsets of $Q$.

Proof. Let $\mathcal{R}$ be a proper nonzero annihilator right ideal of $Q$. Then $\mathcal{R}=\operatorname{rann}(S)$ for some non-empty subset $S$ of $Q$. Note that $S \neq\{0\}$ as $\mathcal{R} \neq Q$. Since the left ideal $\operatorname{lann}(\mathcal{R})$ contains $S$, it is non-zero. By (3.1) (v), $\mathcal{R}$ is closed, so by Lemma 3.4(ii), $(\mathcal{R}, \operatorname{lann}(\mathcal{R}))$ is a maximal orthogonal pair of $Q$. Therefore, by Theorem 4.3 , $\mathcal{R} \cap \operatorname{lann}(\mathcal{R})$ is a maximal zero product subset of $Q$. We have shown that the map makes sense. Now Lemma 4.2 shows that the map is injective and Theorem 4.3 proves that it is surjective too.

4.5. Recall that $Q$ is a Baer ring if every left annihilator of any subset of $Q$ is generated (as a left ideal) by an idempotent element. If $Q$ is unital then it is known that this definition is left-right symmetric. Note that every simple Artinian ring is a unital Baer ring. 
Corollary 4.6. Let $Q$ be a simple unital Baer ring with nonzero nilpotent elements. Then $S \subset Q$ is a maximal zero product subset if and only if $S=e Q(1-e)$ where $e \neq 0,1$ is a nontrivial idempotent of $Q$. Moreover, if $e_{1}$ and $e_{2}$ are idempotents of $Q$ then $e_{1} Q\left(1-e_{1}\right)=e_{2} Q\left(1-e_{2}\right)$ if and only if $e_{1} e_{2}=e_{2}$ and $e_{2} e_{1}=e_{1}$ (equivalently, $\left.e_{1} Q=e_{2} Q\right)$.

Proof. By Theorem 4.3 and Corollary 4.4, the maximal zero product subsets of $Q$ are the intersections $\mathcal{R} \cap \operatorname{lann}(\mathcal{R})$ where $\mathcal{R}$ runs over all proper nonzero annihilator right ideals of $Q$. Since $Q$ is Baer, $\mathcal{R}=e Q$ for some idempotent $e$. Then $\operatorname{lann}(\mathcal{R})=\operatorname{lann}(e Q)=$ $Q(1-e)$. Indeed, one has $a \in \operatorname{lann}(e Q)$ if and only if $a e=0$, or equivalently, $a=$ $a(1-e) \in Q(1-e)$. It is also clear that

$$
\mathcal{R} \cap \operatorname{lann}(\mathcal{R})=e Q \cap Q(1-e)=e Q(1-e) .
$$

Finally, by Corollary 4.4, $e_{1} Q\left(1-e_{1}\right)=e_{2} Q\left(1-e_{2}\right)$ if and only if $e_{1} Q=e_{2} Q$. It is easy to see that the latter condition is equivalent to $e_{1} e_{2}=e_{2}$ and $e_{2} e_{1}=e_{1}$.

4.7. By [8, IV.8], a ring $Q$ is simple with minimal one-sided ideals if and only $Q \cong$ $Y \otimes_{\Delta} X$, where $(X, Y,\langle\cdot, \cdot\rangle)$ is a pair of dual vectors spaces over a division ring $\Delta$, and where the product is given by

$$
\left(y_{1} \otimes x_{1}\right)\left(y_{2} \otimes x_{2}\right)=y_{1} \otimes\left\langle x_{1}, y_{2}\right\rangle x_{2}
$$

for all $x_{1}, x_{2} \in X, y_{1}, y_{2} \in Y$. According to this representation of $Q$, we have (see [8, IV.16.Theorem 1]:

(i) The map $W \mapsto W \otimes X$ is a lattice isomorphism of the lattice $\mathcal{S}(Y)$ of all subspaces of $Y$ onto the lattice $\mathcal{J}_{r}(Q)$ of all right ideals of $Q$.

(ii) The map $V \mapsto Y \otimes V$ is a lattice isomorphism of the lattice $\mathcal{S}(X)$ of all subspaces of $X$ onto the lattice $\mathrm{J}_{l}(Q)$ of all left ideals of $Q$.

4.8. It is easy to check that if $\mathcal{R}=W \otimes X$ is a right ideal of $Q$, then $\operatorname{lann}(\mathcal{R})=Y \otimes W^{\perp}$, where $W^{\perp}=\{x \in X:\langle x, W\rangle=0\}$. Similarly, for any left ideal $\mathcal{L}=Y \otimes V$ of $Q$, $\operatorname{rann}(Y \otimes V)=V^{\perp} \otimes X$. Thus $\mathcal{R}$ is an annihilator right ideal if and only $\mathcal{R}=W \otimes Y$, where $W$ is a closed subspace of $Y$, i.e., $W^{\perp \perp}=W$.

We have a Galois connection between the lattice $\mathcal{S}(X)$ of all subspaces of $X$ and the lattice $\mathcal{S}(Y)$ of all subspaces of $Y$ given by $V \rightarrow V^{\perp}$ and $W \rightarrow W^{\perp}$.

It is easy to see that all finite dimensional subspaces of $X$ (resp. $Y$ ) are closed. Indeed, let $V$ be a finite-dimensional subspace of $X$ with basis $\left\{x_{1}, \ldots, x_{n}\right\}$. Fix a dual set $\left\{y_{1}, \ldots, y_{n}\right\}$ of vectors of $Y$ such that $\left\langle x_{i}, y_{j}\right\rangle=\delta_{i j}, 1 \leq i, j \leq n$, and denote 
$W=\operatorname{span}\left\{y_{1}, \ldots, y_{n}\right\}$. Then we have $X=V \oplus W^{\perp}$ and $Y=W \oplus V^{\perp}$. Clearly, $V \subseteq V^{\perp \perp}$. Thus, $V \neq V^{\perp \perp}$ if and only if there exists a non-zero $u \in V^{\perp \perp} \cap W^{\perp}$. But we have $\langle u, Y\rangle \subseteq\langle u, W\rangle+\left\langle u, V^{\perp}\right\rangle=0$, which implies, by nondegeneracy, that $u=0$.

Corollary 4.9. Let $Q=Y \otimes_{\Delta} X$ be a simple ring with minimal one-sided ideals. Then the map $W \mapsto W \otimes W^{\perp}$ is a bijection from the set of nonzero proper closed subspaces of $Y$ onto the set of maximal zero product subsets of $Q$.

Proof. By Corollary 4.6, any maximal zero product subset $S$ of $Q$ is of the form $S=$ $\mathcal{R} \cap \operatorname{lann}(\mathcal{R})$ for a unique proper nonzero annihilator right ideal $\mathcal{R}$ of $Q$. Now it follows from (4.7) and (4.8) that $\mathcal{R}=W \otimes X$ for a unique nonzero proper closed subspace $W$ of $Y$ and $\operatorname{lann}(W \otimes X)=Y \otimes W^{\perp}$. Hence

$$
S=\mathcal{R} \cap \operatorname{lann}(\mathcal{R})=(W \otimes X) \cap\left(Y \otimes W^{\perp}\right)=W \otimes W^{\perp}
$$

as required.

We finish with an application to the Lie inner ideal structure of simple rings.

4.10. Recall that every associative $\operatorname{ring} Q$ becomes a Lie $\operatorname{ring} Q^{(-)}$under the product $[x, y]=x y-y x$. An additive subgroup $B$ of a Lie ring $L$ is called an inner ideal if $[[B, L], B] \subseteq B$. An inner ideal $B$ is said to be abelian if $[B, B]=0$. An inner ideal $B$ of $Q^{(-)}$is said to be Jordan-Lie if $B^{2}=0$, see [6, 2]. Inner ideals were first systematically studied by Benkart [3], see also [2, 4, 5, 7] for some recent development.

Corollary 4.11. Let $Q$ be a simple associative ring. For an additive subgroup $B$ of $Q$ the following conditions are equivalent.

(i) $B$ is a maximal zero product subset of $Q$.

(ii) $B$ is a maximal regular inner ideal of $Q$.

(iii) $B$ is a maximal Jordan-Lie inner ideal of $Q^{(-)}$.

Moreover, if in addition, $Q$ is not unital (i.e. doesn't contain an identity element) and $\operatorname{char}(Q) \neq 2,3$ then the conditions (i)-(iii) are equivalent to

(iv) $B$ is a maximal abelian inner ideal of $Q^{(-)}$.

Proof. (i) $\Leftrightarrow$ (ii). This is proved in Theorem 4.3 ,

(ii) $\Leftrightarrow$ (iii). Suppose $B$ is a maximal regular inner ideal of $Q$. Then by definition, $B^{2}=0$, so $B$ is Jordan-Lie. Note that any Jordan-Lie inner ideal of $Q$ is contained in a maximal zero product subset of $Q$, which is a maximal regular inner ideal by Theorem 
4.3. Thus, $B$ is maximal as Jordan-Lie. Conversely, if $B$ is a maximal Jordan-Lie inner ideal of $Q$, then it must be maximal regular by above.

Suppose now that $Q$ is not unital.

(iii) $\Leftrightarrow$ (iv). If $B$ is a Jordan-Lie inner ideal of $Q$, then $[B, B] \subseteq B^{2}=0$, so $B$ is abelian. On the other hand, by [6, Theorem 5.4] (applied to the case of a non-unital simple ring), every abelian inner ideal of $Q$ is Jordan-Lie.

\section{REFERENCES}

[1] A.A. Baranov, J. Rowley, Inner ideals of simple locally finite Lie algebras, J. Algebra 379 (2013), $11-30$.

[2] A.A. Baranov, H. Shlaka, Jordan-Lie inner ideals of finite dimensional associative algebras, J. Pure Appl. Algebra (2019), https://doi.org/10.1016/j.jpaa.2019.07.011

[3] G. Benkart, The Lie inner structure of associative rings, J. Algebra 43 (1976) 561-584.

[4] G. Benkart, A. Fernández López, The Lie inner ideal structure of associative rings revisited, Communications in Algebra 37 (2009), 3833-3850.

[5] J. Brox, A. Fernández López, M. Gómez Lozano, Inner ideals of Lie algebras of skew elements of prime rings with involution, Proc. AMS 144 (2016), 2741-2751.

[6] A. Fernández López, Lie inner ideals are nearly Jordan inner ideals, Proc. AMS 142 (2014), 795804 .

[7] A. Fernández López, Jordan Structures in Lie Algebras, Mathematical Surveys and Monographs Vol 240, AMS, 2019.

[8] N. Jacobson, Structure of Rings. Amer. Math. Soc. Colloquium Publ., Providence, 1964.

Department of Mathematics, University of Leicester, Leicester LE1 7RH, UK

E-mail address: ab155@le.ac.uk

Departamento de Álgebra, Geometría y Topología, Universidad de Málaga, 29071, MÁlaga, Spain

E-mail address: emalfer@uma.es 